\title{
WHAT CAUSES THE LOW BINARY FREQUENCY IN THE ORION NEBULA CLUSTER?
}

\begin{abstract}
R. Köhler ${ }^{1}$
RESUMEN

Reportamos los resultados de un censo de binarias en la parte externa del Cúmulo de la Nebulosa de Orión, entre 0.7 y 2 pc del centro del cúmulo. Los resultados serán útiles para decidir si la tasa de formación de binarias fue menor en Orión que en Taurus-Auriga, o si se formaron inicialmente muchas binarias que después fueron destruídas por encuentros estelares cercanos. Encontramos que la frecuencia de binarias entre estrellas de baja masa no depende de la distancia al centro del cúmulo. La frecuencia de compañeras para las estrellas de masa grande tiende a decrecer con el radio, pero el significado estadístico de esta tendencia es pequeño.
\end{abstract}

\section{ABSTRACT}

We report on the results of a binary survey in the outer parts of the Orion Nebula Cluster, 0.7 to $2 \mathrm{pc}$ from the cluster center. The results should help to decide if the binary formation rate was lower in Orion than in TaurusAuriga, or if many binaries formed initially, but were destroyed in close stellar encounters. We find that the binary frequency of low-mass stars does not depend on the distance to the cluster center. The companion star frequency of intermediate- to high-mass stars shows a trend to decrease with cluster radius, but the statistical significance of this trend is rather weak.

\section{Key Words: BINARIES: VISUAL — INFRARED: STARS - STARS: PRE-MAIN SEQUENCE - TECHNIQUES: HIGH ANGULAR RESOLUTION}

\section{INTRODUCTION}

Stellar multiplicity is very common among young solar-like stars, with companion star frequencies close to $100 \%$ for young stars in well-known nearby star-forming $\mathrm{T}$ associations (Leinert et al. 1993; Ghez, Neugebauer \& Matthews 1993; see Duchêne 1999 for a review). Therefore, our current understanding of star formation is that all or nearly all stars form in binary or multiple systems. However, the multiplicity of solar-type main-sequence stars is significantly lower, only about $55 \%$ (Duquennoy \& Mayor 1991). On the other hand, high binary frequencies are not observed among low-mass stars in stellar clusters. Binary surveys in the center of the young Trapezium Cluster (e.g. Prosser et al. 1994; Petr et al. 1998; see McCaughrean 2001 for an overview), which is the core of the Orion Nebula Cluster (ONC), and in the young cluster IC 348 (Duchêne, Bouvier \& Simon 1999), as well as those in older ZAMS clusters (Bouvier, Rigaut \& Nadeau 1997; Patience et al. 1998) show binary fractions that are comparable to that of main-sequence stars, i.e. lower by factors of $2-3$ than those found in loose $\mathrm{T}$ associations.

There are two prevailing theoretical explanations for this discrepancy: Either the initial binary

\footnotetext{
${ }^{1}$ MPI für Astronomie, Heidelberg, Germany.
}

fraction depends on the environmental conditions (Durisen \& Sterzik 1994), i.e., only a relatively small fraction of the stars in Orion formed in binaries. The second theory is based on the disruption of binaries through close stellar encounters (Kroupa 1995; Kroupa, Petr \& McCaughrean 1999). In this case the formation rate of binaries in Orion was as high as in Taurus-Auriga, but many of the binaries were destroyed later in the dense core of the cluster.

In order to obtain observational support for one or the other proposed explanation, we carried out a multiplicity survey of stars in the outskirts of the ONC, about 0.7 to 2 parsec from the cluster center. At these distances from the center, the timescales for stellar interactions are hundreds of times the age of the cluster. Therefore, the binary fraction in the outer parts of the ONC is unlikely to have been modified by the dynamical evolution of the cluster, and should be the intrinsic value resulting from the fragmentation process.

The results presented here are still preliminary; a full analysis will be published elsewhere.

\section{OBSERVATIONS AND DATA REDUCTION}

Since the binaries we are interested in have subarcsecond separations, we decided to use Adaptive Optics (AO) to search for companion stars. AO systems need a relatively bright $\left(\mathrm{I}<12.5^{\mathrm{m}}\right)$ star within 


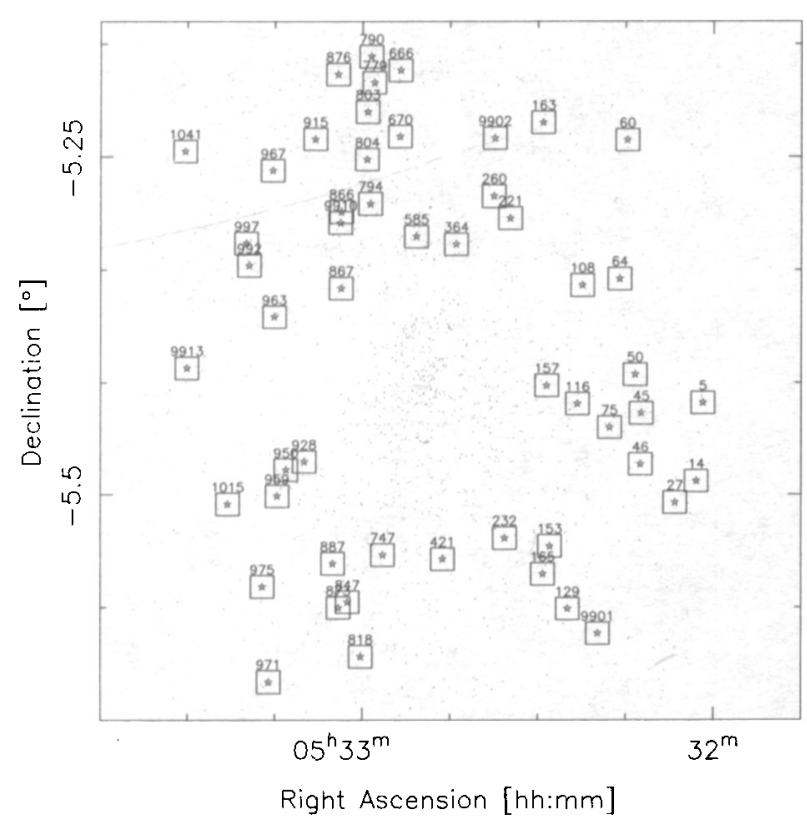

Fig. 1. Fields in the ONC observed for this work. The numbers are the designations in Jones \& Walker (1988) for the stars used to guide the AO system, the dots mark all the stars in their catalog.

about $30^{\prime \prime}$ from the target. We selected a list of stars from Jones \& Walker (1988) at radii of $5^{\prime}$ $15^{\prime}(0.7-2 \mathrm{pc})$ from the cluster center that fulfill this criterion (see Fig. 1). Then we used the TwoMicron All-Sky Survey (2MASS) point source catalog (http://www.ipac.caltech.edu/2mass/) to find stars within $30^{\prime \prime}$ from these guide stars. Figure 2 shows the field around the star JW0971 as an example, Figure 3 shows one of our AO-corrected images of this field.

The larger part of the observations was carried out in three nights in December 2001 at the ESO $3.6 \mathrm{~m}$ telescope on La Silla. We used the adaptive optics system ADONIS with the SHARP II camera to obtain nearly-diffraction limited images in the $\mathrm{K}_{\boldsymbol{s}^{-}}$ band. In February 2002, eleven fields were observed in one half night at the Keck II telescope. We used the adaptive optics system (Wizinowich et al. 2000), and the NIRC 2 camera with the $\mathrm{K}_{p}$ filter.

The data were reduced following standard infrared-imaging techniques, i.e., sky subtraction, flat-fielding and bad-pixel correction. We then used DAOPHOT within IRAF to find stars in the resulting images. Most companion stars are too close to their primaries to be discovered by automatic procedures; they were found by visual inspection.

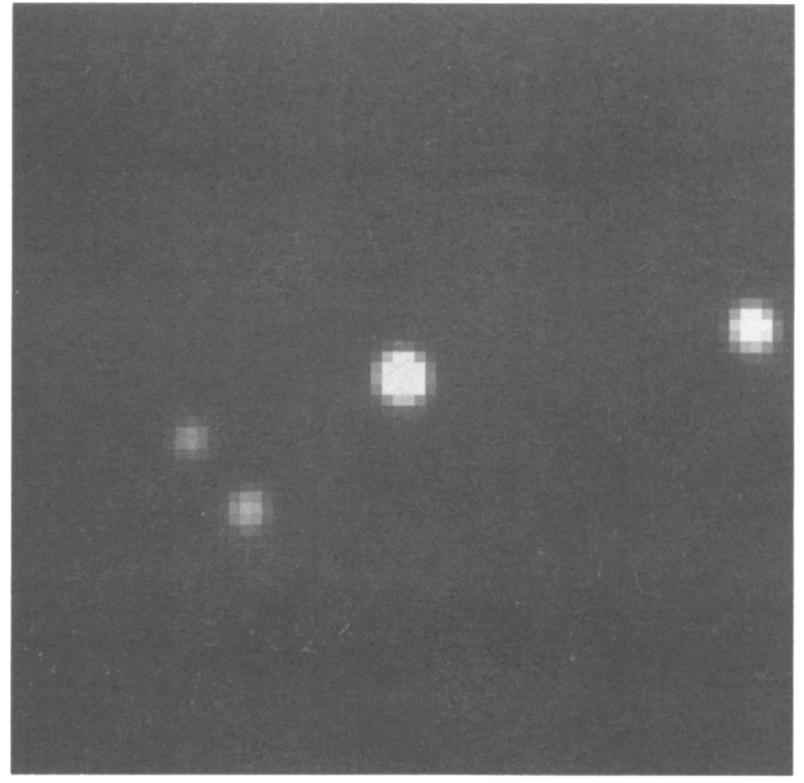

Fig. 2. Quicklook image from the 2MASS database of the region around JW0971. The field of view is about $70^{\prime \prime} \times 70^{\prime \prime}$. North is up and East to the left.

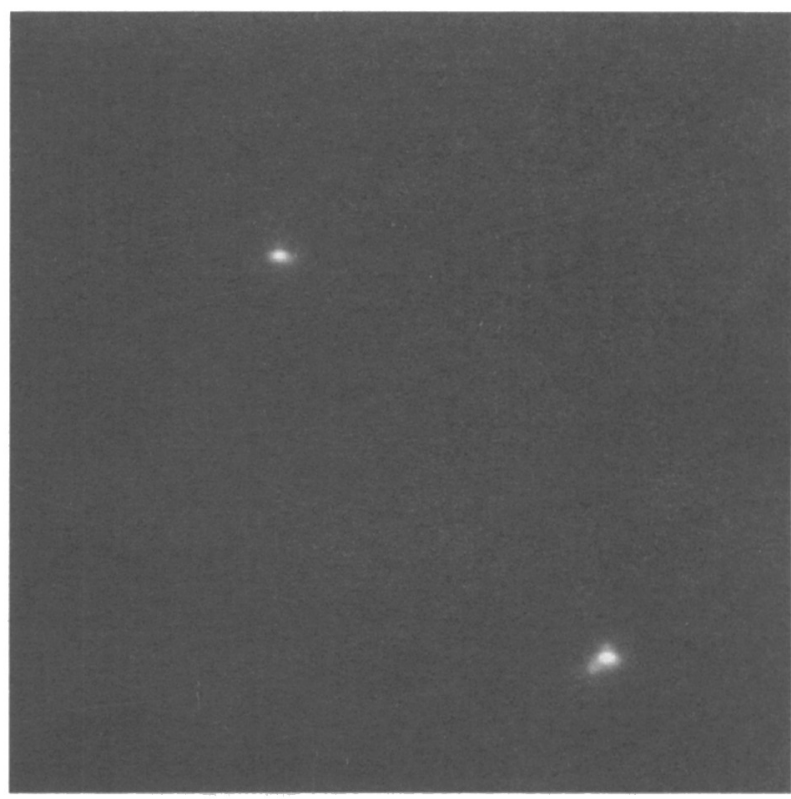

Fig. 3. AO-corrected image of the two stars southeast of JW0971. The star to the southwest has a companion at $0.3^{\prime \prime}$ separation. The field of view is about $12^{\prime \prime} \times 12^{\prime \prime}$.

\section{RESULTS}

In total, we observed 229 stars in 52 fields (see Fig. 1). We are sensitive to companions at separations in the range $0^{\prime \prime} 13-1^{\prime \prime} 11$ or $60-500 \mathrm{AU}$. The lower limit is the diffraction limit of the $3.6 \mathrm{~m}$ telescope in $\mathrm{K}$; the outer limit was chosen to limit the number of chance alignments of unrelated stars. 


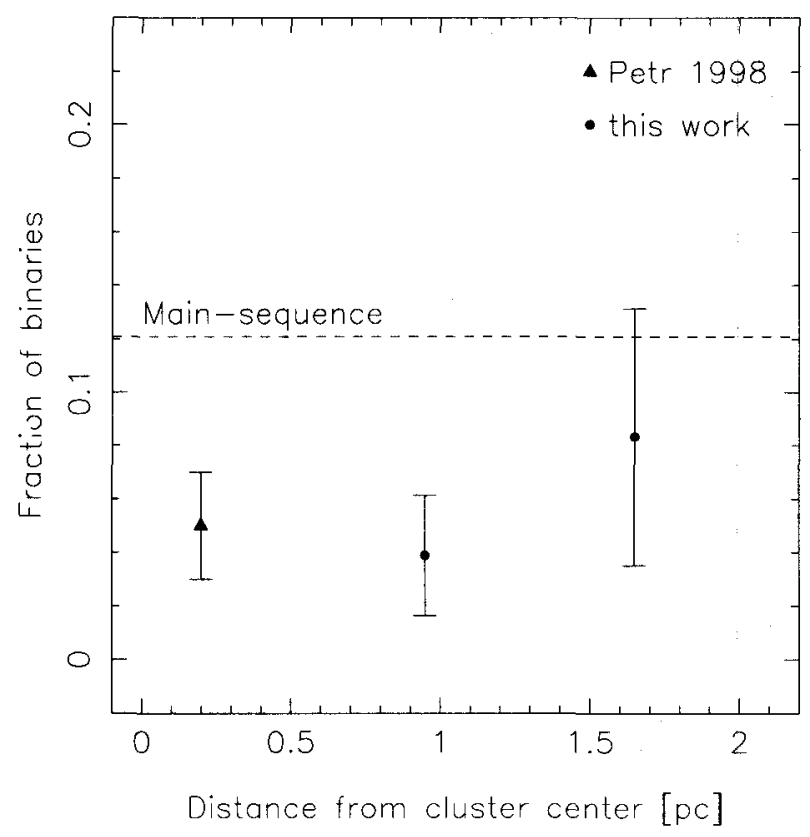

Fig. 4. Frequency of binaries among low-mass stars (mass range $0.1-1.4 \mathrm{M}_{\odot}$ ) as function of the distance to the cluster center. The dashed line shows the companion star frequency among solar-type main-sequence stars (Duquennoy \& Mayor 1991).

These limits are identical to those used by Petr et al. (1998) for their binary survey in the center of the ONC.

We obtained masses for many of our targets from Hillenbrand (1997), and mass estimates based on infrared colors from 2MASS. According to these sources, we have 113 low-mass systems (in the mass range $0.1-1.4 \mathrm{M}_{\odot}$ ), with 88 masses from Hillenbrand (1997) and 25 from 2MASS. Six of these stars have companions, which is equal to $5.3 \pm 2.2 \%$. This is not significantly higher than the result of Petr et al. (1998), who found $5 \pm 2 \%$ binaries among lowmass stars in the cluster core. Figure 4 shows the binary frequency at three distances from the cluster center. There is no significant tendency for a higher binary frequency in the outer parts of the cluster.

Our sample contains 40 systems with masses or mass estimates larger than $1.4 \mathrm{M}_{\odot}$ (35 from Hillenbrand 1997, 5 from 2MASS). However, there is only one star with a mass of over $10 \mathrm{M}_{\odot}$, the lower limit of the high-mass subsample in Petr et al. (1998), which makes a comparison with this high-mass sample meaningless. Instead, we went back to the original data in Petr (1998) and counted intermediateto high-mass stars in their target list. There are 17 systems with four companions (two binaries, one triple) at separations in the range $0 . \prime 13-1{ }^{\prime \prime} 11$, i.e. a

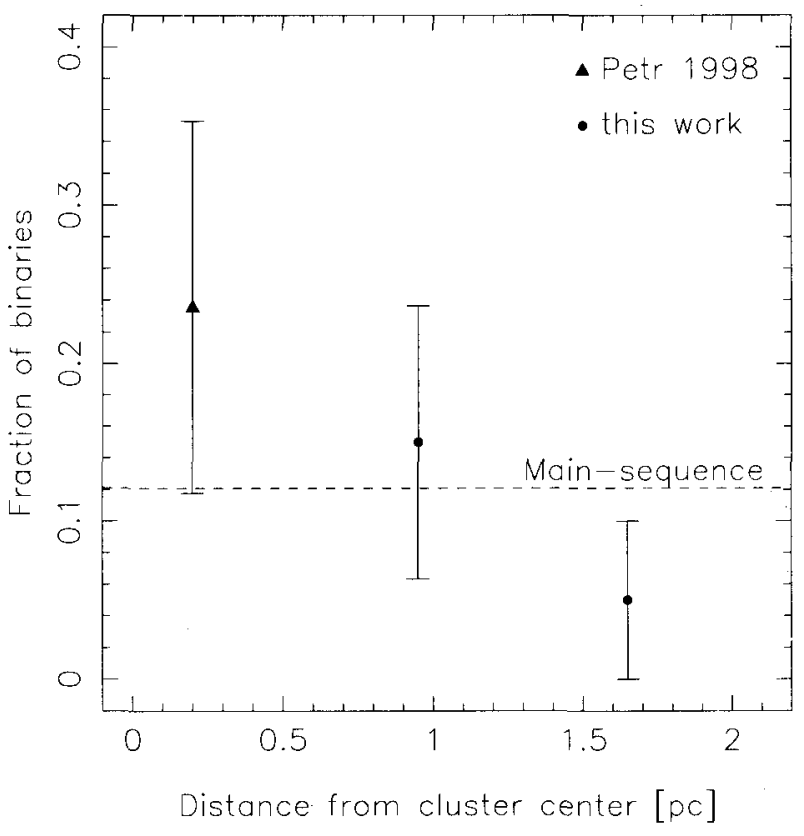

Fig. 5. Frequency of binaries among intermediate to high-mass stars (masses $>1.4 \mathrm{M}_{\odot}$ ) as function of the distance to the cluster center. The dashed line shows the companion star frequency among solar-type (i.e. lowmass) main-sequence stars (Duquennoy \& Mayor 1991).

companion star frequency of $24 \pm 12 \%$. In the outer parts of the cluster, we find 4 companions, which yields a companion star frequency of $10 \pm 5 \%$. Figure 5 shows the results as a function of the distance from the cluster center. Although the companion star frequencies in the cluster core and the outskirts differ by a factor of more than 2, the errors are large, so this is just barely a $1 \sigma$ result.

\section{CONCLUSIONS}

We find no tendency for the binary frequency of low-mass stars to change with distance from the cluster center. This can be explained in two ways:

- Either the initial formation rate of binaries was much lower in Orion than in Taurus-Auriga, and dynamical disruption played only a minor role,

- or the Orion Nebula Cluster was much denser in the past, so that stars that are now at more than $1 \mathrm{pc}$ distance from the center went through enough close encounters to reduce the companion star frequency to the level observed.

We find that binaries are more frequent among intermediate- to high-mass stars than among lowmass stars. We also find that the companion star frequency of intermediate- to high-mass stars tends to decrease with distance from the cluster center. 
However, this result is hardly statistically significant due to the small sample sizes.

I wish to thank my collaborators on this project, Monika G. Petr-Gotzens, Mark McCaughrean, Jerome Bouvier, Gaspard Duchêne, and Andreas Quirrenbach. I also have to thank Friedrich vom Stein since Hans Zinnecker thinks that I always do. This work is based on observations obtained at the European Southern Observatory, La Silla, proposal number 68.C-0539, and at the W.M. Keck Observatory, which is operated as a scientific partnership among the California Institute of Technology, the University of California and the National Aeronautics and Space Administration. The Observatory was made possible by the generous financial support of the W.M. Keck Foundation. The author wishes to recognize and acknowledge the very significant cultural role and reverence that the summit of Mauna Kea has always had within the indigenous Hawaiian community. I am most fortunate to have the opportunity to conduct observations from this mountain. This work has been supported in part by the National Science Foundation Science and Technology Center for Adaptive Optics, managed by the University of California at Santa Cruz under cooperative agreement No. AST-9876783, and by the European Commission Research Training Network "The Formation and Evolution of Young Stellar Clusters" (HPRN-CT-2000-00155).

\section{REFERENCES}

Bouvier, J., Rigaut, F., \& Nadeau, D. 1997, A\&A, 323, 139

Duchêne, G. 1999, A\&A, 341, 547

Duchêne, G., Bouvier, J., \& Simon, T. 1999, A\&A, 343, 831

Duquennoy, A., \& Mayor, M. 1991, A\&A, 248, 485

Durisen, R. H., \& Sterzik, M. F. 1994, A\&A, 286, 84

Ghez, A. M., Neugebauer, G., \& Matthews, K. 1993, AJ, 106,2005

Hillenbrand, L. A. 1997, AJ, 113, 1733

Jones, B. F., \& Walker, M. F. 1988, AJ, 95, 1755

Kroupa, P. 1995, MNRAS, 277, 1491

Kroupa, P., Petr, M., \& McCaughrean, M. 1999, New Astronomy Reviews, 4, 495

Leinert, Ch., Zinnecker, H., Weitzel, N., Christou, J., Ridgway, S. T., Jameson, R., Haas, M., \& Lenzen, R. 1993, A\&A, 278, 129

McCaughrean, M. J. 2001, in The Formation of Binary Stars, proc. IAU Symp. No. 200, eds. H. Zinnecker \& R. D. Mathieu (San Francisco: ASP), 169

Patience, J., Ghez, A. M., Reid, I. N., Weinberger, A. J., \& Matthews, K. 1998, AJ, 115, 1972

Petr, M. G. 1998, PhD Thesis, University of Heidelberg

Petr, M. G., Coudé du Foresto, V., Beckwith, S. V. W., Richichi, A., \& McCaughrean, M. J. 1998, ApJ, 500, 825

Prosser, C. F., Stauffer, J. R., Hartmann, L., Soderblom, D. R., Jones, B. F., Werner, M. W., \& McCaughrean, M. J. 1994, ApJ, 421, 517

Wizinowich, P., Acton, D. S., Lai, O., Gathright, J., Lupton, W., \& Stomski, P. 2000, Proc. SPIE, 4007, 2

Rainer Köhler: Max-Planck-Institut für Astronomie, Königstuhl 17, 69117 Heidelberg, Germany (koehler@ mpia.de). 


\section{DISCUSSION}

Sterzik - What are the masses/spectral types of the "higher mass sample" and how do they compare to the Petr et al. samples?

Köhler - The stars in our "higher mass sample" have an average mass of about $3 M_{\text {sun }}$. The Petr et al. sample consists only of $\mathrm{O}$ and B-stars. So it is possible that we do not see a dependence of multiplicity on radius, but on primary mass.

$A b t$ - Did I understand you to say that there are no short-period binaries in this cluster?

Köhler - We used adaptive-optics observations to search for binaries. This means that we are limited by diffraction limit of the telescope $(0.13 \mathrm{arcsec})$. We cannot find binaries with smaller separations/shorter periods. I am sure there are short-period binaries in the cluster, but we just cannot find them with our survey.

Kaper - That at least one massive binary has been disrupted in the past $(\sim 2.4 \mathrm{Myr}$ age $)$ is demonstrated by the two OB runaway stars $\mu \mathrm{Col}$ and AE Aur that originate in the Trapezium clusters.

Köhler - I am sure disruption of binaries plays a role in the trapezium cluster. The question we are trying to answer is whether it is the dominant process that causes the low binary frequency compared to other star-forming regions.

Clarke - I think that destruction of binaries at these separations can only have happened if the cluster was much denser in the past. In this case you would not expect a star's present location to reflect its conditions at birth and one would not expect a gradient in binary fraction. This hypothesis can only be tested through proper motion experiments that do not factor out any net expansion of the cluster.

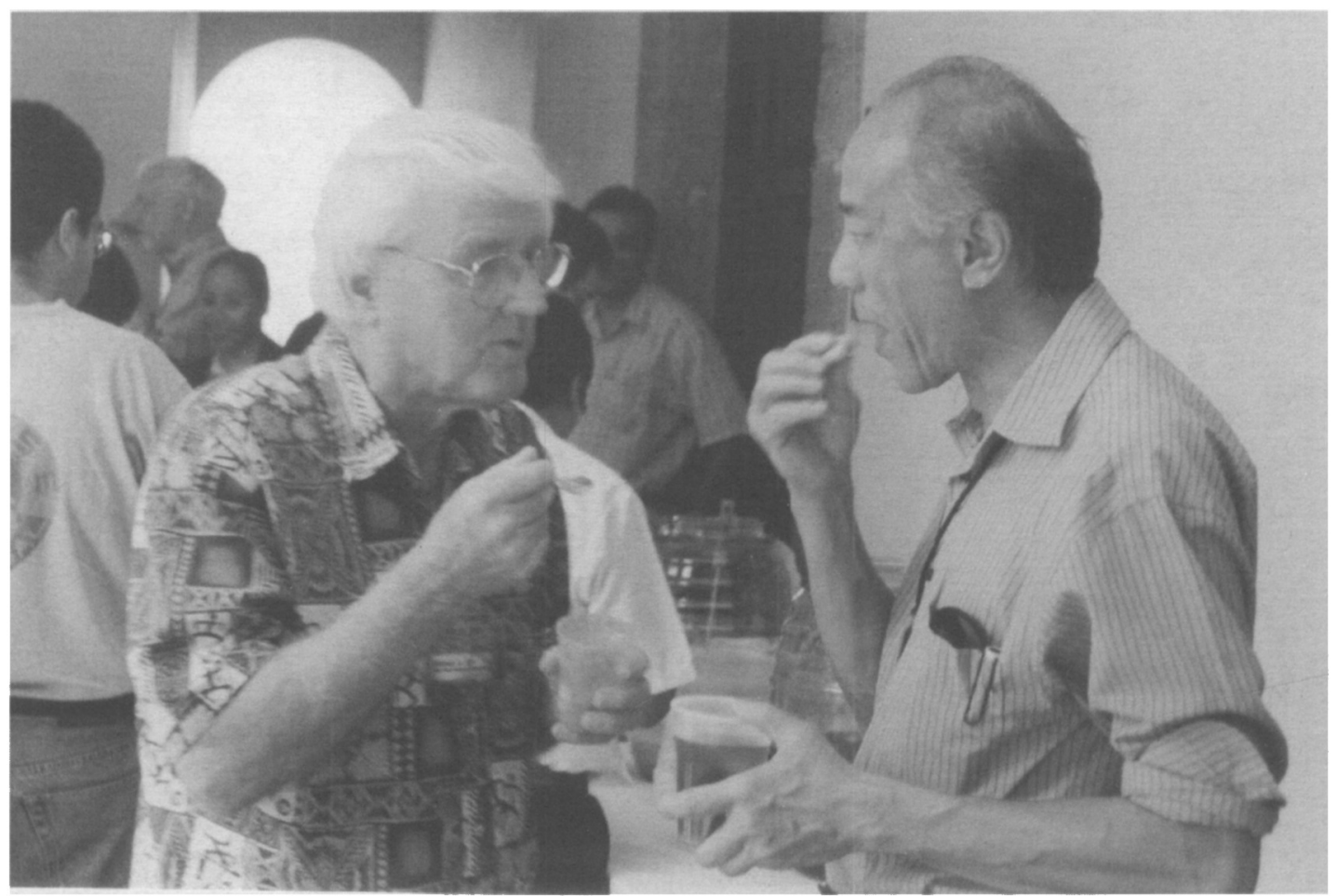

Helmut Abt and Kam-Ching Leung. 